\title{
Dynamics of Non-equilibrium Short-wave-length Phonons in Semiconductor Heterostructures
}

\author{
D. A. ROMANOV, J. EIZENKOP and V. V. MITIN* \\ Wayne State University, Detroit, MI 48202, USA
}

\begin{abstract}
The behavior of short-wave-length acoustic phonons in the vicinity of a quantum well in a GaAs/AlGaAs heterostructure has been investigated. Hot two-dimensional electrons in the well produce longitudinal optical phonons, which decay into almost monoenergetic short-wave-length longitudinal acoustic (LA) phonons. The latter undergo elastic scattering and down-conversion into transverse acoustic (TA) phonons. The distribution of the LA and TA phonons over frequency and distance to the well have been found by solving semi-analytically a system of two kinetic equations with nontrivial boundary conditions and nonlinear dispersion. The distribution functions have essentially non-temperature form even at substantial distance from the well.
\end{abstract}

Keywords: Short-wave-length phonons; Distribution functions; Energy transfer

\section{MOTIVATION AND OBJECTIVES}

The charge carriers heating in the conducting channel of a semiconductor heterostructure device is mainly controlled by the phonon system feedback. The feedback effects, in their turn, are determined by spatial, energy, and modal distributions of the acoustic phonons transferring the excessive lattice energy. In all the existing devicesimulation packages, these contributions are characterized by temperature and/or difference between electron and lattice temperature been taken into account. The energy dissipation from the device operation area is then described by the thermoconductivity equations or in the modified framework of non-local heat transfer [1]. We show that this approach fails under high rate of phonon generation in the device operation channel. We obtain the spatial and energy distribution of the phonons in steady state, and demonstrate their drastically non-temperature form.

\section{THE MODEL}

In a typical situation of high-rate input power, the charge carriers transfer their excessive energy to the phonon system via emission of longitudinal

*Corresponding author. Tel.: (313) 577 8944, Fax: (313) 577 1101, e-mail: mitin@ece6.eng.wayne.edu 
optical phonons of frequency $\omega_{0}$ [1]. These latter are converted into longitudinal acoustic (LA) phonons of frequency $\sim \omega_{0} / 2$ within the conducting layer [2]. The subsequent propagation of the daughter LA phonons away from the layer is assisted by their elastic scattering and anharmonic down-conversion into transverse acoustic (TA) phonons.

The energy range of our interest lies lower then $\omega_{0} / 2$, which corresponds to wave numbers of LA phonons being quite far from the boundary of the Brillouin zone. Thus, we assume the LA phonons dispersion to be linear. For the TA phonons, we model the dispersion curve with simple analytic function representing its wide plateau. The dimensionless dispersion curves are

$$
\omega_{l}=q / \alpha ; \quad \omega_{t}=\tanh q,
$$

where $\alpha=0.59$ is the transverse-to-longitudinal velocity ratio, the frequency unit is the edge frequency of the TA phonon, $\omega_{t}=1.48 \times 10^{13} \mathrm{~s}^{-1}$, and the wavenumber unit is $k_{0}=4.43 \times 10^{7} \mathrm{sm}^{-1}$, the fitting parameters for GaAs. We have used these model dispersion laws in calculating the rates of elastic scattering and anharmonic processes and in deriving and solving the system of kinetic equations for LA and TA phonons.

\section{ELASTIC SCATTERING AND ANHARMONIC DECAY}

The elastic scattering of both LA and TA phonons is GaAs is mostly due to fluctuations of atomic mass of $\mathrm{Ga}$ isotopes, Ga-69(60\% abundance ) and Ga-71 ( $40 \%$ abundance). We have calculated the scattering rates, following the procedure suggested in [3], and implementing our model dispersion laws.

In isotropic approximation, the energy and momentum conservation laws allow two decay processes for LA phonon:

$$
L A(q) \rightarrow L A\left(q^{\prime}\right)+T A\left(q^{\prime \prime}\right)
$$

and

$$
L A(q) \rightarrow T A\left(q^{\prime}\right)+T A\left(q^{\prime \prime}\right)
$$

provided $\mathbf{q}=\mathbf{q}^{\prime}+\mathbf{q}^{\prime \prime}$. The TA mode is non-decaying in this approximation. In the framework of the Fermi golden rule, the decay rates of the processes are expressed in terms of matrix elements of the three-phonon Hamiltonian. We used the thirdorder anharmonic Hamiltonian of the theory of elasticity [4] but assumed the relations between frequencies and wave vectors of the phonons to be given by the model dispersion laws (1).

The obtained elastic scattering rates and anharmonic decay rates are greatly influenced by the huge density of states of the short-wave-length TA phonons. As a result, the processes ending in TA states strongly prevail. In particular, we can neglect the process of Eq. (2).

\section{THE SYSTEM OF KINETIC EQUATIONS}

Making use of the model dispersion laws (1) and the obtained scattering and decay rates, we write the steady-state coupled kinetic equations for the LA and TA phonon distribution functions, $n_{t}(\omega, \theta, x)$ and $n_{t}(\omega, \theta, x)$, in the form:

$$
\begin{aligned}
\frac{\cos \theta}{\alpha} \frac{\omega_{t}}{k_{0}} \frac{\partial n_{l}}{\partial x}= & -\left(\gamma_{l}+\gamma_{t}+\Gamma_{l t t}\right) n_{l} \\
& +\gamma_{l} \bar{n}_{l}+\gamma_{t} \bar{n}_{t} ; \\
\left(1-\omega^{2}\right) \cos \theta \frac{\omega_{t}}{k_{0}} \frac{\partial n_{t}}{\partial x}= & -\left(\gamma_{l}+\gamma_{t}\right) n_{t}+\gamma_{l} \bar{n}_{l} \\
& +\gamma_{t} \bar{n}_{t}+I\left\{n_{l}\right\} .
\end{aligned}
$$

Here, $x$ is the distance to the quantum well, $\theta$ is the angle between the phonon wavevector and the $x$ axis, the bar denotes the function averaged over the angle $\theta$. The scattering rates for the elastic processes ending in LA states and in TA states are

$$
\gamma_{l}=\pi G_{e} \alpha^{3} \omega^{4} \quad \text { and } \quad \gamma_{t}=G_{e} \frac{\alpha^{2} \omega^{2}}{1-\omega^{2}} K^{2}(\omega),
$$


where $G_{e}=2.24 \times 10^{7} \mathrm{~s}^{-1}$, and the auxiliary function $K(\omega)$ is given as

$$
K(\omega)=\frac{1}{2 \alpha} \ln \left(\frac{1+\omega}{1-\omega}\right)
$$

The rate of the only anharmonic process which matters, that of Eq. (3), is

$$
\begin{aligned}
\Gamma_{l t t}= & \frac{\pi G_{a} \alpha^{5}}{8} \int_{0}^{1} d \omega^{\prime} \\
& \frac{\left(\left(\alpha^{2} \omega^{2}-K^{2}\left(\omega^{\prime}\right)-K^{2}\left(\omega-\omega^{\prime}\right)\right)^{2}-2.96 K^{2}\left(\omega^{\prime}\right) K^{2}\left(\omega-\omega^{\prime}\right)\right)^{2}}{\omega^{\prime}\left(\omega-\omega^{\prime}\right)\left(1-\left(\omega-\omega^{\prime}\right)^{2}\right)\left(1-\omega^{2}\right) K\left(\omega^{\prime}\right)} \\
& \times \Theta\left(\omega^{\prime}-\omega+\frac{\omega^{\prime}+\tanh (\alpha \omega)}{1+\omega^{\prime} \tanh (\alpha \omega)}\right) \\
& \times \Theta\left(\omega^{\prime}-\omega-\left|\frac{\tanh (\alpha \omega)-\omega^{\prime}}{1-\omega^{\prime} \tanh (\alpha \omega)}\right|\right) .
\end{aligned}
$$

The functional $I\left\{n_{l}\right\}$ describing an anharmonic generation of the TA phonons in the process of Eq. (3) is given by the expression

$$
\begin{aligned}
I\left\{n_{l}\right\}= & \frac{\alpha G_{a}}{2 \omega K^{2}(\omega)} \int_{0}^{\pi} \sin \theta^{\prime} d \theta^{\prime} \\
& \int d \omega^{\prime} \frac{\omega^{\prime 3} n_{l}\left(\omega^{\prime}, \theta^{\prime}, x\right)}{\left(\omega-\omega^{\prime}\right) K^{2}\left(\omega^{\prime}-\omega\right)\left(1-\left(\omega-\omega^{\prime}\right)^{2}\right)} \\
& \times\left(\left(\omega^{\prime 2}-K^{2}\left(\omega^{\prime}-\omega\right)-K^{2}(\omega)\right)^{2}-2.96 K^{2}(\omega)\right. \\
& \left.\times K^{2}\left(\omega^{\prime}-\omega\right)\right)^{2}\left(S\left(\omega, \omega^{\prime}, \theta, \theta^{\prime}\right)\right)^{-1 / 2} \Theta\left(S\left(\omega, \omega^{\prime}, \theta, \theta^{\prime}\right)\right) .
\end{aligned}
$$

In these expressions, $G_{a}=1.1 \times 10^{8} \mathrm{~s}^{-1}$, and

$$
\begin{aligned}
S\left(\omega, \omega^{\prime}, \theta, \theta^{\prime}\right)= & \left(2 \omega^{\prime} K(\omega)\right)^{2}\left(1-\cos ^{2} \theta-\cos ^{2} \theta^{\prime}\right) \\
& -4 \omega^{\prime} K(\omega) \cos ^{2} \theta \cos ^{2} \theta^{\prime} \\
& \times\left(K^{2}\left(\omega^{\prime}-\omega\right)-\omega^{\prime 2}-K^{2}(\omega)\right) \\
& -\left(K^{2}\left(\omega^{\prime}-\omega\right)-\omega^{\prime 2}-K^{2}(\omega)\right)^{2} .
\end{aligned}
$$

In Eqs. (4), we neglect the LA-TA and TA-TA nonlinear terms ensuing from the process (3). This linearization is well justified by the fact that even in the highly non-equilibrium situation that we consider the numbers of TA phonons (and LA phonons at frequencies less than $\omega_{t}$ ) in individual q-states are much less than unity. Note also that the functions $n_{l}(\omega, \theta, x)$ and $n_{t}(\omega, \theta, x)$ incorporate the densities of states, i.e., they directly give the phonon density.

\section{BOUNDARY CONDITIONS}

The boundary conditions for the functions $n_{l}$ and $n_{t}$ have a non-trivial form in this case. First, for zero-temperature sink, situated at $\mathrm{x}=\mathrm{L}$ and being ideally adhesive for the incoming phonons, the boundary conditions read,

$$
n_{l}(L, \omega, \theta)=0 ; \quad n_{l}(L, \omega, \theta)=0 \quad \text { for } \cos \theta<0 .
$$

The boundary conditions at $\mathrm{x}=0$ are more complicated, because this plane not only reflects both LA and TA phonons that arrive to this plane from inside the bulk but also generates LA phonons. In the case of specular reflection, for the TA phonon distribution function we have

$$
n_{t}(0, \omega, \theta)=n_{t}(0, \omega, \pi-\theta) .
$$

To obtain a boundary condition for the LA distribution function, we suppose that LA phonons are being generated in a plane situated in the substrate at small distance, $\Delta x$, from the boundary. This corresponds to a generation term proportional to $\delta(x-\Delta x)$ in the first of Eqs. (4). The generated LA phonons blow isotropically from the generating plane, and half of them undergo reflection from the boundary. Integrating the first of the kinetic Eqs. (4) through the generating plane, and passing to the limit $\Delta x \rightarrow 0$, we obtain the boundary condition for the LA phonon distribution function,

$$
\begin{aligned}
& \cos \theta \cdot\left(n_{l}(0, \omega, \theta)-n_{l}(0, \omega, \pi-\theta)\right) \\
& \quad=2 G_{0} \frac{\alpha k_{0}}{\omega_{t}} \delta\left(\omega-\omega_{0} / 2\right),
\end{aligned}
$$

where $G_{0}$ is the generation rate of LA phonons in the quantum well. This boundary condition imposes highly anisotropic form of the phonon distribution functions in the substrate. 


\section{NUMERICAL SOLUTIONS}

From the kinetic Eqs. (4) and the boundary conditions (8)-(10), we have found analytic expressions for the angular dependencies of the LA and TA phonon distribution functions in terms of the angle-averaged functions, $\bar{n}_{l}(\omega, x)$ and $\bar{n}_{t}(\omega, x)$. Making use of these expressions and integrating the kinetic equations over the angle $\theta$, we come to a close system of integral equations for the averaged distribution functions.

We have solved this system numerically, using the weighted Picard iteration procedure. In doing so, we had to overcome substantial difficulties in calculating three- and five-dimensional integrals. The Romberg integration method was used for successive calculation of these multidimensional integrals [5]. The spatial and frequency dependencies of the LA and TA distribution functions were obtained for particular case of $\mathrm{GaAs} / \mathrm{AlGaAs}$ hesterostructure. The electron gas in the conducting channel has the denisty of $10^{12} \mathrm{~cm}^{-2}$ and is subject to an electric field of $10^{3} \mathrm{~V} / \mathrm{cm}$ intensity (these parameters correspond to the intial LA phonons production rate of $10^{12} \mathrm{~cm}^{-2} \mathrm{~s}^{-1}$. The obtained results are presented in Figures 1 and 2.

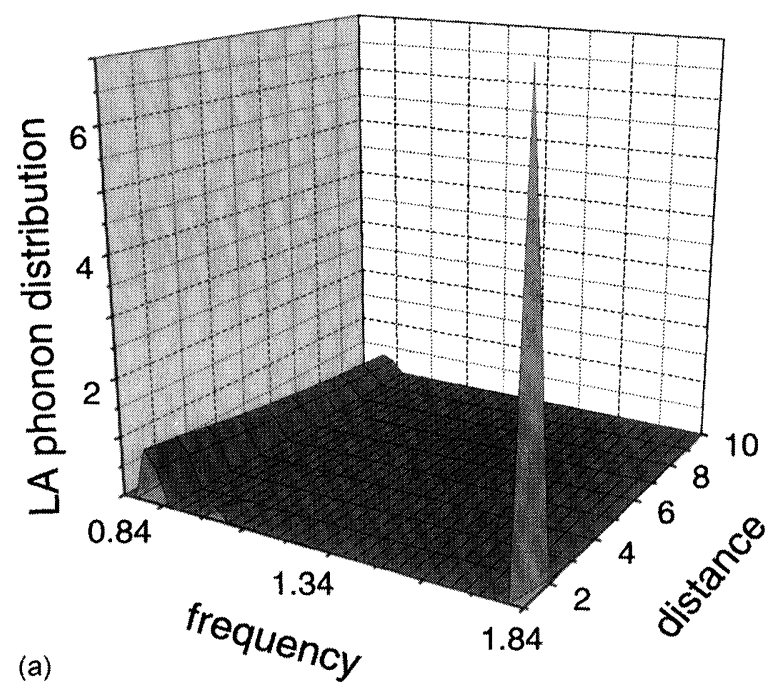

FIGURE 1a The distribution function of high-frequency LA phonons in the substrate, in units of $4 \times 10^{19} \mathrm{~cm}^{-3}$. The frequency is in units of $\omega_{\mathrm{t}}$, the distance scale is $1 \mu \mathrm{m}$.

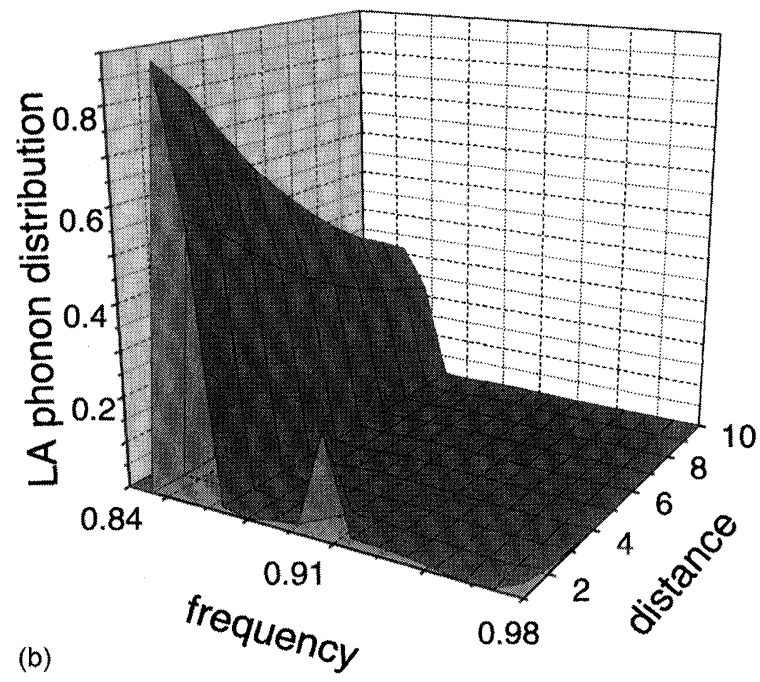

FIGURE 1b The same function in detail, at the frequencies near $\omega_{\mathrm{t}}$.

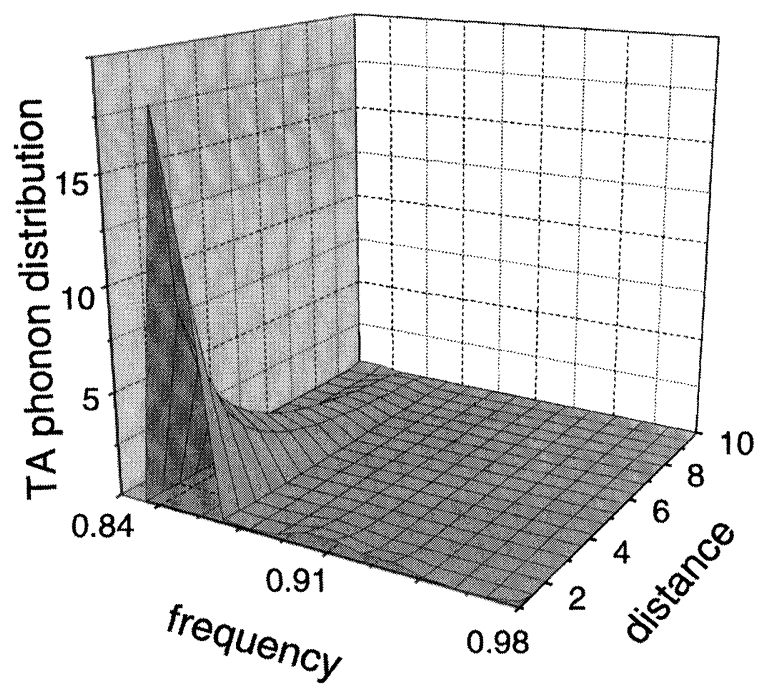

FIGURE 2 The distribution function of TA phonons at frequencies near $\omega_{t}$; the notations are the same as in Figure 1.

\section{DISCUSSION AND CONCLUSIONS}

The Figures show the phonon distributions in the substrate as functions of frequency and the distance from the conducting channel. These distribution functions are essentially determined by a complicated interplay of elastic scattering and 
anharmonic decay rates, both of which substantially depend on phonon energy. The key processes of down-conversion are controlled by anharmonicity of the lattice vibrations, the elastic scattering is mainly due to isotopic impurities. The LA phonons undergo anharmonic decay preferably into TA modes. The non-decaying phonons of TA branch convert into LA phonons (which can decay into TA phonon of lower frequencies) only by the elastic scattering on impurities (the first term in the right-hand side of the second equation in (4)). Thus, the excess energy removal from the device operation channel proceeds through the cascade phonon down-conversions, assisted by the interbranch elastic scattering. These processes start in high-frequency range, where the TA phonon dispersion curve is almost flat. This flattening corresponds to giant density of states, which states are thereby strongly preferable final states for scattering and decay processes. As the result, the distribution functions have considerable peaks in the vicinity of the frequency $\omega_{t}$, which corresponds to TA flattening. The peaks cannot be simply ascribed to the factor of density of states in the functions $\omega_{l}(\omega, \theta, x)$ and $\omega_{t}(\omega, \theta, x)$ : the form of the TA peak is quite different from that of the density of TA states, while the density of LA states has no peak at all at these frequencies. These distribution peaks persist through distances of several micrometers.

The obtained results convincingly prove that most part of the excessive energy of the acoustic phonon system has non-temperature form, and pose a challenge to incorporate this non-temperature behavior in forthcoming generations of nanoscale-device-simulation packages.

\section{Acknowledgments}

We thank Dr. B. A. Glavin for stimulating discussions. Financial support by U.S. Army Research Office is gratefully acknowledged.

\section{References}

[1] Hot Carriers in Semiconductor Nanostructures: Physics and Applications, Edited by Shah, J. Boston: Academic Press, 1992.

[2] Romanov, D. A., Glavin, B. A., Mitin, V. V. and Stroscio, M. A. (1999). "Stimulated decay of non-selectively pumped optical phonons in GaAs", Phys. Rev. B, 60, 4771-4777.

[3] Maris, H. J. and Tamura, S. (1993). "Anharmonic decay and propagation of phonons", Phys. Rev. B, 47, 727.

[4] Tucker, J. W. and Rampton, V. W. (1972). Microwave Ultrasonics in Solid State Physics, Amsterdam: NorthHolland.

[5] Press, W. H., Vettering, W. T., Teukolsky, S. A. and Flannery, B. P. (1988). Numerical Recipes in C, Cambridge: University press. 

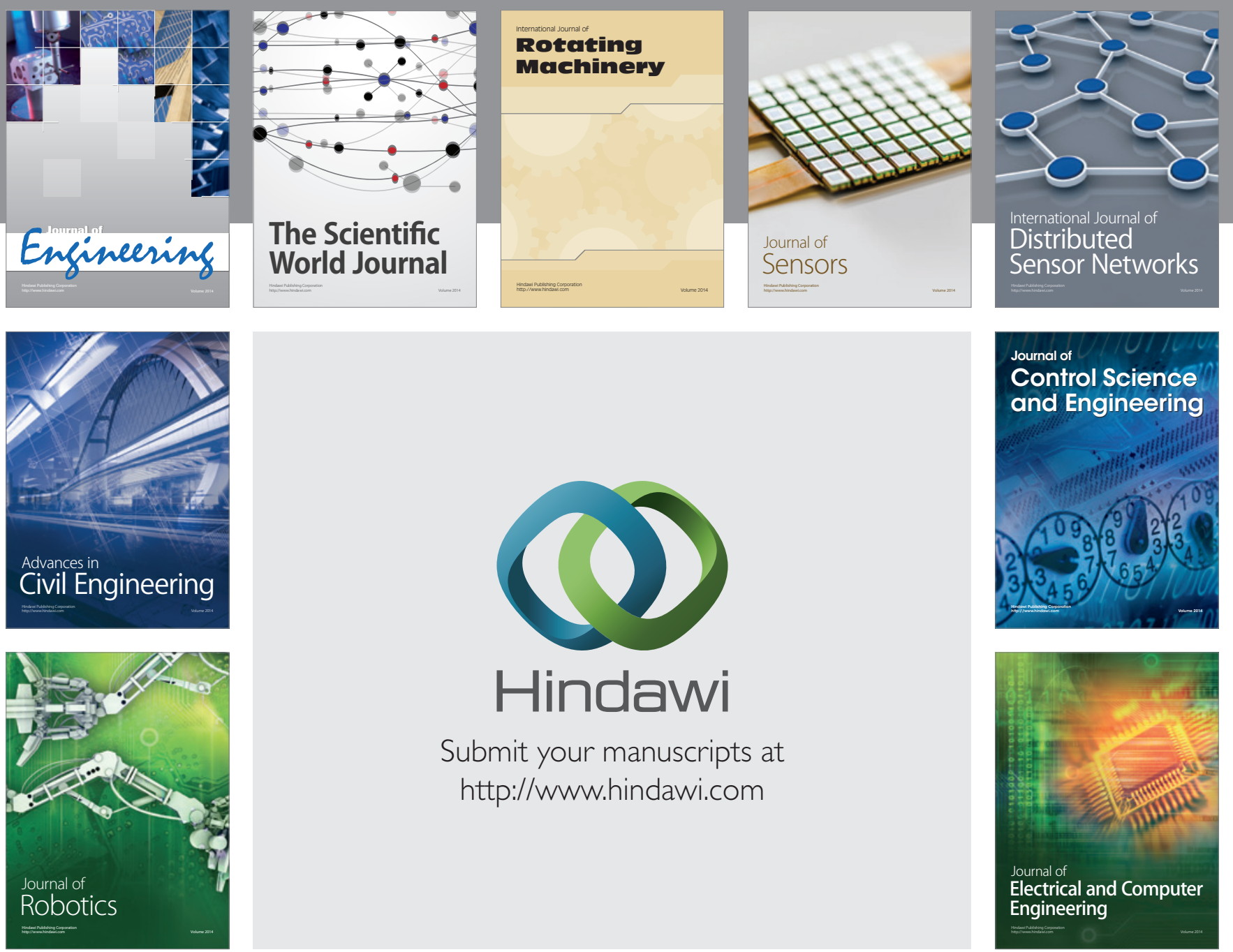

Submit your manuscripts at

http://www.hindawi.com
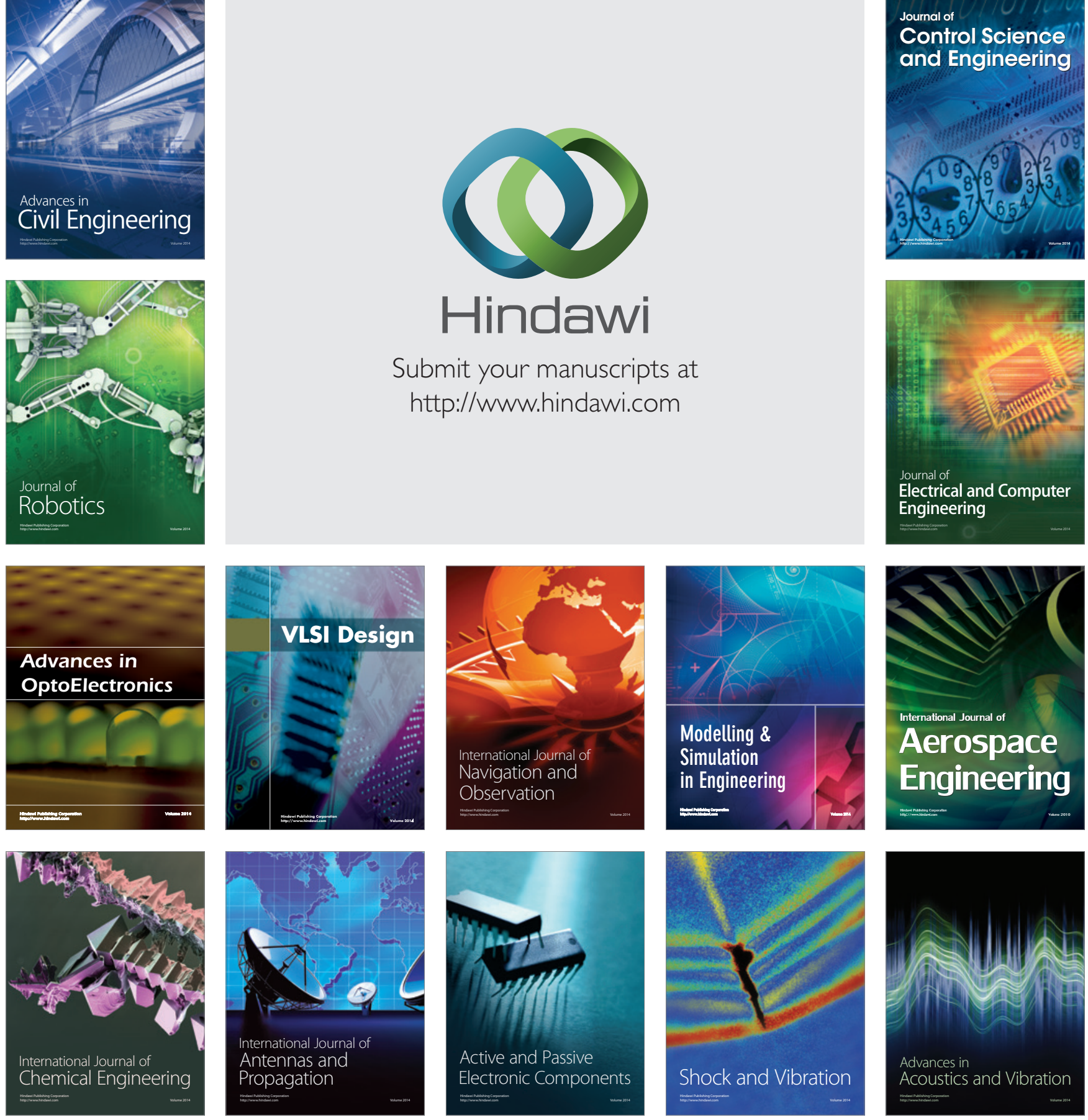